\title{
Semeadoras-adubadoras em semeadura convencional de soja
}

\author{
Fertilizer-seeders in the soybean conventional sowing
}

\author{
Maria Cristina Cavalheiro Tourino ${ }^{\mathrm{I}}$ Pedro Milanez de Rezende ${ }^{\mathrm{II}}$ Lucas Alves da Silva ${ }^{\text {III }}$ \\ Luis Gustavo Pessôa de Almeida ${ }^{I V}$
}

\begin{abstract}
- NOTA -
RESUMO

O objetivo deste estudo foi avaliar o desempenho de três semeadoras-adubadora, em solo preparado convencionalmente e cinco densidades de plantas nas linhas. Foram avaliadas a produtividade e outras características agronômicas da soja. A semeadura foi realizada nos anos 2001/2002 e 2002/2003, com as cultivares 'Monarca'e 'Conquista'. Foram utilizadas semeadoras adubadoras, sendo duas de precisão e uma de fluxo contínuo e uma semeadura manual (testemunha). O delineamento experimental foi o de blocos ao acaso, com três repetições, e tratamentos em parcelas subdivididas. Foi realizada a avaliação da uniformidade de espaçamento entre plantas nas linhas, produtividade de grãos, dos legumes por planta, da massa de 100 grãos, da altura das

agronomic characteristics. The sowing was carried through in years 2001/2002 and 2002/2003, with Monarca and Conquista cultivars. Fertilizer-seeders were used, being two precision seeders and a one seed drills and manual sowing (witness). The experimental design was randomized blocks, with three replications, and treatments in split plots. Accuracy in plant spacing in the row, grains yield, pods for plant, mass of 100 grains, plants height, lodging and real density of plants in the row had been evaluated. The precision seeder with vacuum metering system provided larger accuracy and grains yield, in relation the mechanical metering system, in the densities from 10 to 19 plants $\mathrm{m}^{-1}$; in the 22 plants $\mathrm{m}^{-1}$ density it did not have difference between the precision seeders with regard to yield; the seed drills, with the conformation used in this work, was not adjusted for the implantation of the soybean crop.
\end{abstract} plantas, do acamamento e da densidade real de plantas nas linhas. A semeadora de precisão com dosador pneumático a vácuo foi mais uniforme e proporcionou maior produtividade de grãos em relação à semeadora de dosador de tipo mecânico, nas densidades de 10 a 19 plantas $\mathrm{m}^{-1}$; na densidade de 22 plantas $m^{-1}$, não houve diferença entre as semeadoras de precisão com relação à produtividade; a semeadora de fluxo contínuo, com a conformação utilizada neste trabalho, não foi adequada para a implantação da cultura da soja.

Palavras-chave: Glycine max, produtividade, precisão, acamamento, densidade real de plantas.

\section{ABSTRACT}

The objective of this study was to evaluate the effect of three fertilizer-seeders, in ground conventionally prepared and five densities of plants in the row in soybean yield and
Key words: Glycine max, yield, precision, lodging, stand.

O aumento da produtividade das culturas é uma meta buscada por produtores e pesquisadores. Para alcançar essa meta, um dos principais fatores envolvidos é o estabelecimento de um estande com plantas uniformemente distribuídas nas linhas, utilizando com eficiência os recursos disponíveis no ambiente. De acordo com RAMBO et al. (2003), a melhor distribuição de plantas na área pode contribuir para o aumento da produtividade, pois permite o melhor aproveitamento da água, da luz e dos nutrientes disponíveis no solo (VENTIMIGLIA et al., 1999; TOURINO et al. 2002).

\footnotetext{
IDepartamento de Engenharia, Universidade Federal de Lavras (UFLA), CP 37, 37200-000, Lavras, MG, Brasil. E-mail: mcctouri@ufla.br. Autor para correspondência.

IIDepartamento de Agricultura, UFLA, Lavras, MG, Brasil.

IIICurso de Engenharia Agrícola, UFLA, Lavras, MG, Brasil.

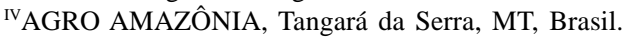


Para a implantação da cultura da soja, existem vários tipos de semeadoras, com diversos sistemas para a dosagem de sementes. Dentre os mais utilizados, estão os sistemas mecânicos com discos perfurados horizontais e os sistemas pneumáticos. Teoricamente, sistemas pneumáticos podem proporcionar o estabelecimento de estandes mais uniformes e, conseqüentemente, maior produtividade. No Brasil, entretanto, existem poucos estudos, que relacionam o uso dessas máquinas com o estabelecimento de estandes uniformes e a produtividade da soja.

Neste trabalho, foi avaliado o desempenho em campo, sob semeadura convencional, de três semeadoras com diferentes sistemas de dosagem, mais uma testemunha semeada manualmente, em cinco densidades, sobre a produtividade de grãos e outras características agronômicas da soja.

As avaliações foram realizadas nos anos agrícolas 2001/2002 e 2002/2003, na Fazenda Palmital, da Fundação de Apoio ao Ensino, à Pesquisa e à Extensão (FAEPE), município de Ijaci, MInas Gerais, (MG), latitude $21^{\circ} 10^{\prime}$ sul, longitude $44^{\circ} 55^{\prime}$ e altitude 805 metros, em uma área de Latossolo Vermelho Amarelo Câmbico com aproximadamente um ha. A precipitação total dos meses de novembro a abril foi de $1257,9 \mathrm{~mm}$ ( $1^{\circ}$ ano) e de $1077,9 \mathrm{~mm}$ ( $2^{\circ}$ ano). Não foram observados períodos com deficiência hídrica durante o desenvolvimento da cultura. As médias de umidade relativa e temperatura foram de $76 \%$ e $22,2^{\circ} \mathrm{C}$ ( $1^{\circ}$ ano) e de $75 \%$ e $22,6^{\circ} \mathrm{C}$ ( $2^{\circ}$ ano).

As cultivares de soja utilizadas foram 'Monarca' (1ํano) e ‘Conquista' (MG/BR 46) ( $2^{\circ}$ ano), ambas indicadas para o Estado de Minas Gerais. As sementes foram inoculadas com Bradyrhizobium japonicum, na proporção de $0,25 \mathrm{~kg}$ de inoculante para $50 \mathrm{~kg}$ de sementes. Posteriormente, elas foram lubrificadas com grafite. $\mathrm{O}$ fertilizante NPK utilizado foi o granulado de fórmula 4-30-10, na dosagem de 400 $\mathrm{kg} \mathrm{ha}^{-1}$.

As máquinas avaliadas foram duas semeadoras de precisão (uma com dosador de sementes do tipo pneumático a vácuo - semeadora A - e outra com dosador de sementes do tipo mecânico, de discos horizontais - semeadora B) e uma semeadora de fluxo contínuo (dosador de sementes do tipo rotor acanalado helicoidal - semeadora C - avaliada somente no primeiro ano). Nas semeadoras, foram utilizados discos com 108 células (A) e 90 furos (B). Na semeadora C, as lingüetas de restrição foram reguladas na posição central. Todas as máquinas foram utilizadas na versão para uso em preparo convencional, com sulcadores para sementes do tipo discos duplos e fertilizantes discos duplos defasados (A) e hastes (B). Na semeadora C, o sulco para fertilizantes foi realizado pelo mesmo conjunto sulcador de sementes. O sistema de fechamento e compactação dos sulcos foi composto por rodas duplas inclinadas (A); rodas planas com borracha lisa (B) e correntes (C).

O preparo do solo, nos dois anos, foi realizado de forma convencional, com uma aração e duas gradagens em área com declividade de $2 \%$. A umidade no solo foi considerada adequada para a semeadura da soja nos dois anos. Durante o desenvolvimento da cultura, foram realizadas pulverizações para os controles de plantas invasoras e da lagarta da soja. Nos tratamentos com máquina tratorizada, a semeadura foi realizada em área maior (aproximadamente 1ha), onde as semeadoras (A com 4 linhas e B e C com 5 linhas) efetuaram duas passagens na área (ida e volta), de acordo com os tratamentos, sendo demarcadas, posteriormente, as subparcelas em uma ida ou volta. Todas as subparcelas foram constituídas por quatro linhas espaçadas entre si de $45 \mathrm{~cm}$, com cinco metros de comprimento (área $=9 \mathrm{~m}^{2}$ ). Foram consideradas como área útil apenas duas linhas centrais, escolhidas de forma a não coincidirem com os rastros dos pneus dos tratores, sendo desprezado 0,50 metro nas extremidades (área útil = 3,6 $\mathrm{m}^{2}$ ).

Todas as semeadoras foram reguladas, sendo considerados os seguintes aspectos: a porcentagem de germinação das sementes de $80 \%$, o deslizamento das rodas de acionamento dos dosadores de $10 \%$ (A) e de $5 \%$ (B e C) e o índice de danos mecânicos às sementes de $0 \%$ (A) e $5 \%$ (B e C). As máquinas foram reguladas para uma profundidade de semeadura de $3 \mathrm{~cm}$ e fertilização de $8 \mathrm{~cm}$. A semeadora $\mathrm{C}$, em virtude de suas características, foi regulada para a mesma profundidade de semeadura e fertilização $(3 \mathrm{~cm})$. Para a tração das semeadoras, foram utilizados os tratores Valtra 900L 4x2 TDA, Valmet 88 e Valmet 85, nas velocidades 2,3 $\mathrm{m} \mathrm{s}^{-1}$; $1,8 \mathrm{~m} \mathrm{~s}^{-1} \mathrm{e} 1,6 \mathrm{~m} \mathrm{~s}^{-1}$ - semeadoras A, B e C, respectivamente (1o ano), e $2,1 \mathrm{~m} \mathrm{~s}^{-1}$ e $1,5 \mathrm{~m} \mathrm{~s}^{-1}$ - semeadoras A e B, respectivamente ( $2^{\circ}$ ano). Nos tratamentos manuais, a colocação do fertilizante no solo e a abertura dos sulcos para sementes foram realizadas utilizando-se a semeadora A com os reservatórios de sementes vazios e com as rodas de compactação sem pressão. A semeadura foi realizada com auxílio de réguas perfuradas. Na posição de cada furo, foram colocadas duas sementes. Vinte e cinco dias após a emergência, foi realizado o desbaste de forma a manter somente uma planta em cada posição. O fechamento e a compactação dos sulcos foram realizados manualmente.

O delineamento experimental foi o de blocos ao acaso, com três repetições, em esquema de parcelas subdivididas. Nas parcelas, foram dispostas cinco 
densidades (10, 13, 16, 19 e 22 plantas $\left.\mathrm{m}^{-1}\right)$. Nas subparcelas, foram dispostas as semeadoras mais o tratamento manual. $\mathrm{O}$ fator ano não foi considerado como variável na análise estatística realizada. Na área útil de cada tratamento, foram avaliados: produtividade de grãos, legumes por planta, massa de 100 grãos, altura das plantas, grau de acamamento (BERNARD et al., 1965) e densidade real de plantas nas linhas. Os dados de produtividade e massa de 100 grãos foram corrigidos para $13 \%$ de umidade. A uniformidade de espaçamentos entre plantas nas linhas (U) foi realizada medindo-se os espaçamentos entre 51 plantas consecutivas e avaliada de acordo com os critérios propostos por TOURINO \& KLINGENSTEINER (1983). Os dados de acamamento foram transformados para a análise estatística em $\sqrt{x}$.

Na tabela 1, são apresentados os resultados médios obtidos nos dois anos. A semeadora C apresentou a menor densidade real de plantas nas linhas e, conseqüentemente, a menor uniformidade de espaçamentos entre plantas - menor que 50\% desempenho insatisfatório, segundo TOURINO \& KLINGENSTEINER (1983), não atingindo o estande desejado. Devido à grande produção por planta, a semeadora $\mathrm{C}$ apresentou uma produtividade razoável, entretanto, o baixo número de plantas nas linhas provocou o não fechamento da cultura e, conseqüentemente, uma grande infestação de plantas daninhas, causando ineficiência no seu controle. As plantas produzidas foram baixas, com caules de grande diâmetro. Embora não tenha ocorrido acamamento devido à grande quantidade de ramificações e peso das plantas, alguns legumes ficaram em contato com o solo. A soma desses fatores dificulta a colheita mecanizada, podendo causar perdas, embuchamentos e danos na barra segadora das colhedoras, o que não contribui para a recomendação dessa máquina na implantação da cultura da soja. As causas do baixo estande obtido foram, provavelmente, devidas à imprecisão do sistema de dosagem, colocação de sementes e fertilizantes no mesmo sulco e ausência de compactação das sementes, provocada pelo uso de correntes. Esses resultados diferem dos obtidos, em plantio direto, por PORTELLA et al. (1998): "rotores helicoidais com dimensionamento adequado, poderiam desempenhar a função de discos alveolados com igual nível de precisão”.

Até a densidade de 19 plantas $\mathrm{m}^{-1}$, a maior produtividade foi obtida com a semeadora A, que, dentre as semeadoras, apresentou maior uniformidade de espaçamentos entre plantas, em ambos os anos. O aumento da densidade, entretanto, provocou uma redução da uniformidade desses espaçamentos, ficando no primeiro ano maior que $50 \%$ somente nas densidades de 10 a 16 plantas $\mathrm{m}^{-1}$ e no segundo entre $50 \%$ e $75 \%$ (desempenho apenas regular, segundo TOURINO \& KLINGENSTEINER, 1983). A redução na uniformidade, com o aumento da densidade, pode ser justificada pelo aumento da velocidade tangencial no centro das células dos discos (maior relação de transmissão), o que reduz o tempo de exposição semente/célula e conseqüentemente prejudica a seleção correta das sementes. No caso da semeadora B, por se tratar de um sistema de dosagem mecânico, em que outros fatores, como posicionamento do ponto de liberação das sementes para o tubo condutor e altura de queda destas até o fundo do sulco, também influenciam a uniformidade dos espaçamentos entre plantas, os resultados mostram uma precisão igual e acima de $50 \%$ apenas nas densidades 13 e 19 plantas $\mathrm{m}^{-1}$. Nas demais densidades, os resultados foram insatisfatórios. A redução da uniformidade para a semeadora $A$, entretanto, não provocou perda de produtividade até a densidade de 19 plantas $\mathrm{m}^{-1}$ devido à compensação obtida pelo aumento no número de plantas presentes nas linhas, que contribuíram para o número total de legumes por planta. Com o aumento para 22 plantas $\mathrm{m}^{-1}$, no entanto, a produtividade caiu, e a semeadora B, mesmo apresentando menor uniformidade de espaçamento entre plantas, foi superior. Isso ocorreu porque a densidade real de plantas ficou bem abaixo do desejado para essa máquina, provocando uma maior produção de legumes por planta, o que contribuiu para a maior produtividade obtida. De forma geral, no primeiro ano, a semeadora $B$ apresentou densidade real de plantas nas linhas menor do que a programada.

A semeadura manual apresentou maior uniformidade em relação a todos os tratamentos. Entretanto, essa maior uniformidade não correspondeu uma maior produtividade em relação às máquinas, como era esperado. Isso ocorreu pelo fato de a cobertura das sementes e a compactação nos sulcos de semeadura terem sido realizadas com auxílio de enxadas, o que não contribuiu para a adequada compressão entre sementes e solo úmido, além da maior exposição do sulco durante o seu fechamento. Isso mostra a importância dos componentes das semeadoras responsáveis pela cobertura e compactação do solo em torno das sementes. A produtividade e densidade real, na semeadura manual, se mantiveram em patamares semelhantes nos dois anos, enquanto que para as semeadoras A e B houve uma redução na produtividade no segundo ano. As densidades reais, para as duas máquinas, apesar do uso das mesmas regulagens nos dois anos, aumentaram no segundo ano devido, em parte, devido à redução nas velocidades de 
Tabela 1 - Uniformidade de espaçamentos entre plantas nas linhas (U) e médias dos desdobramentos para produtividade (P), legumes por planta (LPP), massa de 100 grãos (M100), altura de plantas (AP), grau de acamamento (G.A.) e densidade real de plantas nas linhas (Dr), na cultura da soja. Ijaci/MG, 2001/2002 e 2002/2003. ${ }^{(1)}$

\begin{tabular}{|c|c|c|c|c|c|c|c|c|}
\hline $\begin{array}{l}\text { Densidade } \\
\left(\text { plantas } \mathrm{m}^{-1}\right)\end{array}$ & Semeadora $^{(2)}$ & $\mathrm{U}(\%)$ & $\mathrm{P}\left(\mathrm{kg} \mathrm{ha}^{-1}\right)$ & LPP & M100 (g) & $\mathrm{AP}(\mathrm{cm})$ & G.A. & $\begin{array}{c}\text { Dr } \\
\left(\text { plantas } \mathrm{m}^{-1}\right)\end{array}$ \\
\hline & - & ------- & --Ano 1 - C & ar "Mor & "” -----------. & -----------. & ----------- & ----- \\
\hline \multirow[t]{4}{*}{10} & Manual & 90,7 & 2885 b & $62 \mathrm{~b}$ & 17,0 a & 108,5 a & 1,3 a & 9,4 a \\
\hline & A & 70,7 & 3471 a & $60 \mathrm{~b}$ & 17,8 a & 107,8 a & $1,3 \mathrm{a}$ & 10,8 a \\
\hline & $\mathrm{B}$ & 36,7 & 2326 c & $65 \mathrm{~b}$ & $15,5 \mathrm{~b}$ & $82,3 \mathrm{~b}$ & $1,0 \mathrm{a}$ & $7,0 \mathrm{~b}$ \\
\hline & $\mathrm{C}$ & 20,6 & 2008 c & $261 \mathrm{a}$ & $16,8 \mathrm{a}$ & $77,6 \mathrm{~b}$ & $1,0 \mathrm{a}$ & $1,2 \mathrm{c}$ \\
\hline \multirow[t]{4}{*}{13} & Manual & 90,0 & $2929 a b$ & $51 \mathrm{~b}$ & $17,5 \mathrm{a}$ & 109,5 a & $1,6 \mathrm{a}$ & $12,5 \mathrm{a}$ \\
\hline & A & 56,0 & 3490 a & $48 \mathrm{~b}$ & $17,6 \mathrm{a}$ & $102,6 \mathrm{a}$ & $1,0 \mathrm{a}$ & $13,6 \mathrm{a}$ \\
\hline & $\mathrm{B}$ & 46,0 & 3262 a & $69 \mathrm{~b}$ & 17,8 a & 107,2 a & $2,3 \mathrm{~b}$ & $9,5 \mathrm{~b}$ \\
\hline & $\mathrm{C}$ & 8,9 & 2425 b & 199 a & 16,8 a & $84,4 \mathrm{~b}$ & $1,0 \mathrm{a}$ & $1,4 \mathrm{c}$ \\
\hline \multirow[t]{4}{*}{16} & Manual & 88,7 & $2826 \mathrm{~b}$ & $49 \mathrm{~b}$ & $17,0 \mathrm{ab}$ & 109,8 a & $1,6 \mathrm{ab}$ & $15,1 \mathrm{a}$ \\
\hline & A & 50,7 & 3785 a & $39 \mathrm{~b}$ & $18,2 \mathrm{ab}$ & $112,2 \mathrm{a}$ & $1,6 \mathrm{ab}$ & 16,0 a \\
\hline & B & 36,7 & 3409 a & $64 \mathrm{~b}$ & $18,5 \mathrm{~b}$ & 110,7 a & $2,0 \mathrm{~b}$ & $11,5 \mathrm{~b}$ \\
\hline & $\mathrm{C}$ & 15,5 & 1763 c & $191 \mathrm{a}$ & 16,9 a & 87,5 b & $1,0 \mathrm{a}$ & $1,0 \mathrm{c}$ \\
\hline \multirow[t]{4}{*}{19} & Manual & 82,0 & $2572 \mathrm{~b}$ & $48 \mathrm{~b}$ & 16,7 a & 107,6 a & $2,3 \mathrm{a}$ & $18,1 \mathrm{a}$ \\
\hline & A & 46,0 & 3505 a & $41 \mathrm{~b}$ & $18,1 \mathrm{~b}$ & 114,5 a & $1,6 \mathrm{a}$ & 19,4 a \\
\hline & B & 38,0 & 3482 a & $53 \mathrm{~b}$ & $18,1 \mathrm{~b}$ & 111,9 a & $2,0 \mathrm{a}$ & $11,6 \mathrm{~b}$ \\
\hline & $\mathrm{C}$ & 10,2 & $2137 b$ & 258 a & $16,5 \mathrm{a}$ & $74,5 \mathrm{~b}$ & $1,0 \mathrm{~b}$ & $1,2 \mathrm{c}$ \\
\hline \multirow[t]{4}{*}{22} & Manual & 69,3 & $2513 \mathrm{~b}$ & $55 \mathrm{~b}$ & $16,7 a b$ & 110,3 a & 3,0 a & 21,3 a \\
\hline & A & 45,3 & $2797 \mathrm{ab}$ & $31 \mathrm{~b}$ & $16,9 a b$ & 117,6 a & $2,0 \mathrm{~b}$ & $22,2 \mathrm{a}$ \\
\hline & B & 37,3 & 3246 a & $55 \mathrm{~b}$ & $17,8 \mathrm{~b}$ & 106,7 a & $2,3 \mathrm{ab}$ & $15,0 \mathrm{~b}$ \\
\hline & $\mathrm{C}$ & 15,3 & 1419 c & $190 \mathrm{a}$ & 15,9 a & $81,7 \mathrm{~b}$ & $1,0 \mathrm{c}$ & $1,1 \mathrm{c}$ \\
\hline & & & -Ano 2 - Cl & Ir "Conc & 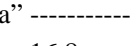 & & & --- \\
\hline \multirow[t]{3}{*}{10} & Manual & 90,7 & 2561 a & $33 \mathrm{~b}$ & 16,8 a & $88,3 \mathrm{~b}$ & $1,0 \mathrm{~b}$ & $9,1 \mathrm{~b}$ \\
\hline & A & 64,0 & 2672 a & $35 \mathrm{~b}$ & 15,8 a & 98,3 a & $1,4 \mathrm{a}$ & $12,0 \mathrm{a}$ \\
\hline & B & 46,7 & 2139 a & $48 \mathrm{a}$ & $14,5 \mathrm{~b}$ & 82,5 c & $1,4 \mathrm{a}$ & $8,7 \mathrm{~b}$ \\
\hline \multirow[t]{3}{*}{13} & Manual & 93,3 & 2512 b & $28 \mathrm{a}$ & 17,3 a & $91,7 \mathrm{~b}$ & $1,0 \mathrm{a}$ & $12,4 \mathrm{~b}$ \\
\hline & A & 69,3 & 3378 a & $34 \mathrm{a}$ & $16,8 \mathrm{a}$ & 99,5 a & $1,0 \mathrm{a}$ & $15,1 \mathrm{a}$ \\
\hline & B & 50,0 & $2681 \mathrm{~b}$ & $34 \mathrm{a}$ & $16,7 \mathrm{a}$ & 94,2 a & $1,3 \mathrm{a}$ & $11,4 \mathrm{~b}$ \\
\hline \multirow[t]{3}{*}{16} & Manual & 90,7 & 2566 a & $26 \mathrm{a}$ & 17,5 a & $91,3 \mathrm{~b}$ & $1,4 \mathrm{~b}$ & $14,9 \mathrm{~b}$ \\
\hline & A & 50,0 & 2883 a & $30 \mathrm{a}$ & $16,4 \mathrm{a}$ & $103,0 \mathrm{a}$ & $1,5 \mathrm{~b}$ & $18,4 \mathrm{a}$ \\
\hline & B & 36,7 & 2715 a & 34 a & 16,9 a & 99,6 a & $2,2 \mathrm{a}$ & $15,4 \mathrm{~b}$ \\
\hline \multirow[t]{3}{*}{19} & Manual & 84,7 & $2748 \mathrm{~b}$ & 29 a & $16,8 \mathrm{a}$ & $93,3 \mathrm{~b}$ & $1,5 \mathrm{a}$ & $17,7 \mathrm{~b}$ \\
\hline & A & 59,3 & 3462 a & 35 a & $17,4 \mathrm{a}$ & $101,8 \mathrm{a}$ & $1,8 \mathrm{a}$ & 21,5 a \\
\hline & B & 56,7 & $3032 a b$ & 26 a & 16,9 a & 98,3 a & $1,8 \mathrm{a}$ & $18,5 \mathrm{~b}$ \\
\hline \multirow[t]{3}{*}{22} & Manual & 84,0 & 2627 a & 20 a & 17,3 a & 96,7 a & $2,0 \mathrm{~b}$ & $22,6 \mathrm{ab}$ \\
\hline & A & 58,7 & 2287 a & $19 \mathrm{a}$ & $17,2 \mathrm{a}$ & 103,2 a & $3,0 \mathrm{a}$ & 24,2 a \\
\hline & B & 42,7 & 2527 a & $25 \mathrm{a}$ & $17,1 \mathrm{a}$ & $97,3 \mathrm{a}$ & $2,8 \mathrm{a}$ & $21,3 \mathrm{~b}$ \\
\hline
\end{tabular}

(1) Médias seguidas pela mesma letra não diferem entre si, ao nível de 5\% de probabilidade, pelo teste de SNK (Student-Newman-Keuls).

(2) Velocidade das semeadoras: $\mathrm{A}-2,3 \mathrm{~m} \mathrm{~s}^{-1} ; \mathrm{B}-1,8 \mathrm{~m} \mathrm{~s}^{-1} ; \mathrm{C}-1,6 \mathrm{~m} \mathrm{~s}^{-1}$ (ano 1); $\mathrm{A}-2,1 \mathrm{~m} \mathrm{~s}^{-1} ; \mathrm{B}-1,5 \mathrm{~m} \mathrm{~s}^{-1}$ (ano 2).

deslocamento das duas semeadoras, que proporcionou uma redução acentuada nos índices médios de falhas destas (21,5\% e 41,1\% no primeiro ano; 11,3\% e 22,4\%, no segundo ano, com conseqüente aumento da uniformidade). A redução no índice de falhas está relacionada com o maior tempo de exposição semente/

Ciência Rural, v.39, n.1, jan-fev, 2009. 
célula obtido pela redução da velocidade das máquinas. O aumento no número de plantas, entretanto, não foi suficiente para compensar a redução na produção por planta, resultando em produtividade menor. Além das diferentes condições ambientais ocorridas naturalmente de um ano para o outro, os diferentes potenciais produtivos das cultivares utilizadas justificam parte desses resultados.

A semeadora de precisão com dosador pneumático a vácuo foi mais uniforme e proporcionou maior produtividade de grãos em relação à semeadora de dosador de tipo mecânico, nas densidades de 10 a 19 plantas $\mathrm{m}^{-1}$; na densidade de 22 plantas $\mathrm{m}^{-1}$, não houve diferença entre as semeadoras de precisão com relação à produtividade; a semeadora de fluxo contínuo, com a conformação utilizada neste trabalho, não foi adequada para a implantação da cultura da soja.

\section{REFERÊNCIAS}

BERNARD, R.L. et al. (Ed.). Results of the cooperative uniform soybean tests. Washington: USDA, 1965. 134p.
PORTELLA, J.A. et al. Regularidade de distribuição de sementes e de fertilizantes de semeadoras para plantio direto de trigo e soja. Engenharia Agrícola, Jaboticabal, v.17, n.4, p.57-64, 1998.

RAMBO, L. et al. Rendimento dos grãos de soja em função do arranjo de plantas. Ciência Rural. Santa Maria, v.33, n.3, p.405-411, 2003.

TOURINO, M.C.C.; KLINGENSTEINER, P. Ensaio e avaliação de semeadoras-adubadoras. In: CONGRESSO BRASILEIRO DE ENGENHARIA AGRÍCOLA, 13., 1983, Seropédica,RJ. Anais... Seropédica: SBEA, 1983. p.103-107.

TOURINO, M.C.C. et al. Espaçamento, densidade e uniformidade de semeadura na produtividade e características agronômicas da soja. Pesquisa Agropecuária Brasileira, Brasília, v.37, n.8, p.1071-1077, 2002.

VENTIMIGLIA, L.A. et al. Potencial de rendimento da soja em razão da disponibilidade de fósforo no solo e dos espaçamentos. Pesquisa Agropecuária Brasileira, Brasília, v.34, n.2, p.195-199, 1999. 